\title{
Atomic exchange processes and bimodal initial growth of $\mathrm{Co} / \mathrm{Cu}(001)$
}

\author{
F. Nouvertné, U. May, M. Bamming, A. Rampe, U. Korte, and G. Güntherodt \\ 2. Physikalisches Institut der RWTH Aachen, D-52056 Aachen, Germany \\ R. Pentcheva and M. Scheffler \\ Fritz-Haber-Institut der Max-Planck-Gesellschaft, Faradayweg 4-6, D-14195 Berlin-Dahlem, Germany
}

(Received 3 August 1999)

\begin{abstract}
The initial growth of $\mathrm{Co}$ on $\mathrm{Cu}(001)$ is atomically identified by scanning tunneling microscopy using $\mathrm{CO}$ titration and density-functional theory total-energy calculations. Both reveal that at low coverage Co adatoms occupy substitutional sites in the $\mathrm{Cu}$ substrate surface that act as pinning centers for subsequent island nucleation. The interaction with diffusing adatoms is found to be attractive and stronger for nearest-neighbor Co compared to $\mathrm{Cu}$. The atomic substitution process actuates a bimodal growth mode as experimentally found in the island size distributions. This gives rise to a high density of small Co islands and large Co-decorated $\mathrm{Cu}$ islands. [S0163-1829(99)08943-2]
\end{abstract}

Growth and characterization of magnetic thin films on nonmagnetic substrates are of considerable interest because of the relevance of the interface morphology for phenomena such as surface magnetic anisotropy, ${ }^{1,2}$ giant magnetoresistance, ${ }^{3-5}$ and interlayer exchange coupling $^{6-8}$ - effects that potentially form the basis of the currently developed "magnetoelectronics." In this context, heteroepitaxy of $\mathrm{Co}$ on $\mathrm{Cu}(001)$ represents a model system for understanding the interplay between structural and magnetic properties 9 and was already the subject of many studies. ${ }^{10-15}$ The small lattice mismatch of the two materials [the lattice constant of (magnetic, fcc) Co is $2 \%$ smaller than that of (nonmagnetic, fcc) $\mathrm{Cu}$ ] and the low miscibility of $\mathrm{Co}$ in bulk $\mathrm{Cu}$ (Ref. 16) are typically considered as a prerequisite for a sharp, good quality interface. Yet, the possibility of surface alloying cannot be ruled out, as it had been observed in other lattice-mismatched heteroepitaxial systems (e.g., Refs. 17-20). For $\mathrm{Co} / \mathrm{Cu}(001)$ an ordered $c(2 \times 2)$-surface alloy was shown to be unstable against phase separation. ${ }^{21}$ For a coverage $\theta \leqslant 2 \mathrm{ML}$, an unexplained bilayer growth mode was reported. ${ }^{10,13,22}$ In a recent scanning tunneling microscopy (STM) study, Fassbender, Allenspach, and Dürig, ${ }^{23}$ found intermixing between $\mathrm{Co}$ and $\mathrm{Cu}$. Co-rich regions in the substrate and copper-rich regions in the top layer were identified via a bias dependent contrast reversal in STM images of 0.12 ML.

In this paper we report on a combined experimental and theoretical study of the surface morphology of Co on $\mathrm{Cu}(001)$ in the submonolayer regime using STM and density-functional theory (DFT) total-energy calculations. In the STM images we are able to resolve single Co atoms incorporated in the substrate. On the basis of the DFT calculations, we give a quantitative picture of the microscopic processes during the initial stages of growth: Some of the deposited $\mathrm{Co}$ atoms exchange sites with atoms of the $\mathrm{Cu}$ substrate surface. This atomic exchange in conjunction with its coverage dependence leads to three different adatom species on the surface: substitutional $\mathrm{Co}$, on-surface $\mathrm{Co}$, and on-surface $\mathrm{Cu}$. The DFT calculations confirm that the substitutional Co adatoms act as nucleation centers, as suggested previously by Fassbender, Allenspach, and Dürig, ${ }^{23}$ and their attractive potential is investigated quantitatively. The influence of these pinning centers on the initial growth is observed in island size distributions for different substrate temperatures, and deviations from standard nucleation theory ${ }^{24}$ are discussed. The two different adatoms diffusing on the surface $(\mathrm{Co}$ and $\mathrm{Cu})$ give rise to a bimodal growth mode with islands of different size and chemical composition as identified in a CO-titration experiment.

The experiments were carried out in a system of ultrahigh vacuum chambers with a base pressure of $10^{-10}$ mbar that provides via a transfer chamber the following facilities: molecular-beam epitaxy (MBE), surface analysis by means of Auger electron spectroscopy (AES), low-energy electron diffraction, and STM using a modified Besocke-type microscope. ${ }^{25}$ The surface of the $\mathrm{Cu}(001)$ single crystal was prepared by standard methods. The Co films were deposited on the $\mathrm{Cu}(001)$ substrate in the MBE chamber by means of an electron-beam evaporator. The evaporation rate, ranging between 0.1 and $0.25 \mathrm{ML} / \mathrm{min}$, was monitored using a calibrated quartz-crystal microbalance.

The DFT calculations were performed with the fullpotential-linearized augmented plane-wave (FP-LAPW) method, ${ }^{26}$ treating the exchange-correlation potential in the local-density approximation (LDA). ${ }^{27}$ Below we focus on surface-energy changes and relative stability of different adsorbate phases. While quantitatively the results are affected by magnetism, we found that the trend is well described by a nonmagnetic calculation. ${ }^{28} \mathrm{We}$ will therefore neglect spin polarization in the present work but will discuss it elsewhere. ${ }^{29}$ The surface was modeled by a slab with five substrate layers, and in the $z$ direction the distance between these periodically repeated slabs was $18 \AA$. For a $(3 \times 3)$-surface unit cell, we used $16 \mathbf{k}_{\|}$points in the surface Brillouin zone, an energy cutoff of $15.6 \mathrm{Ry}$ and a Fourier series expansion of the interstitial electronic density and potential up to $G_{\max }=12.0 \mathrm{bohr}^{-1}$. The wave functions were expanded in spherical harmonics up to $l_{\max }^{\mathrm{wf}}=10$, nonspherical contributions to charge density and potential are considered up to $l_{\max }^{\mathrm{pot}}=4$. 


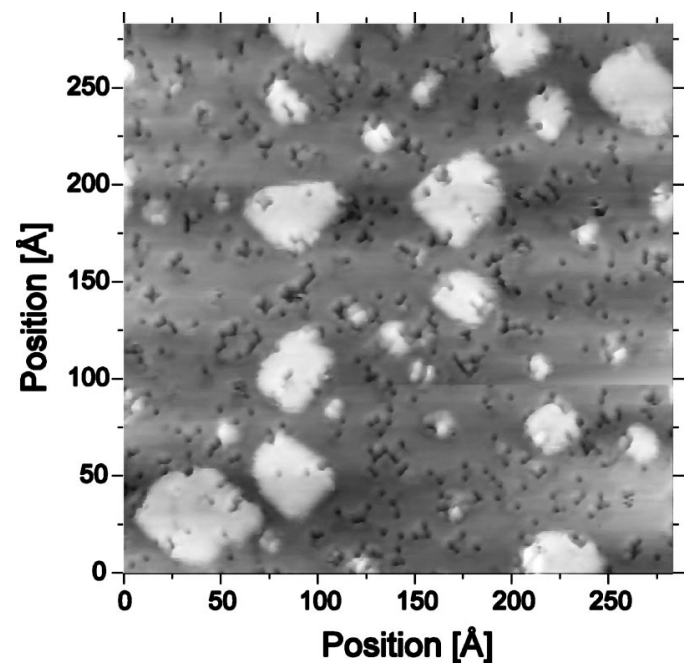

FIG. 1. STM image ( $U_{\text {tip }}=0.9 \mathrm{~V}, I=0.4 \mathrm{nA}$ ) of $0.12 \mathrm{ML}$ Co deposited on $\mathrm{Cu}(001)$ at room temperature. Small indentations (dark gray) are interpreted as incorporated Co atoms (see text).

A STM image for a coverage of 0.12-ML Co deposited on $\mathrm{Cu}(001)$ at room temperature is shown in Fig. 1. The light gray imaged islands of the first layer have an approximately rectangular shape with edges along the [110] and the [1 $\overline{1} 0]$ direction of the substrate. The height of these islands above the substrate is imaged as $1.8 \pm 0.2 \AA$. A striking feature is the small dark indentations in the uncovered gray substrate region as well as in the islands with an imaged depth of $0.6 \pm 0.1 \AA$ with reference to the respective surface. Monoatomic inclusions are clearly visible together with small agglomerates. Even within these agglomerates, the atomic arrangement is resolved. We note that these observations differ from the ones by Fassbender, Allenspach, and Dürig ${ }^{23}$ which found for the same coverage fuzzy islands in the top layer with elongated structures in between.

In order to quantitatively identify the chemical composition of the surface layer, total-energy calculations are used to determine stable and metastable adsorbate configurations. Two different adsorption sites are studied: on surface with a Co adatom on a hollow site on the $\mathrm{Cu}(001)$ surface and substitutional with a $\mathrm{Co}$ atom exchanging with a $\mathrm{Cu}$ atom in the top substrate layer (Co-sub). Corresponding to the STM experiment the calculations were carried out for a $0.11-\mathrm{ML}$ Co coverage in a $(3 \times 3)$-surface unit cell. The adsorption geometries are given in Fig. 2.

Epitaxial growth is a nonequilibrium situation. However, if the deposition rate is not too high, structures in local thermal equilibrium can evolve. For this reason we determine stable and metastable geometries with respect to the adsorption energy per adatom,

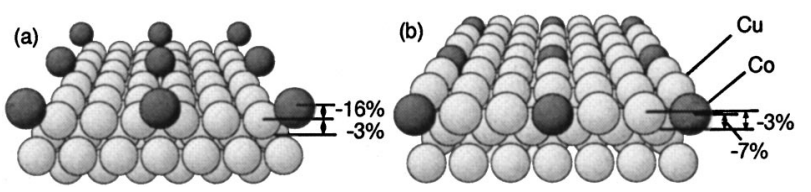

FIG. 2. $\mathrm{Cu}(001)-(3 \times 3)$-Co structure with $\mathrm{Co}$ adatoms at (a) on-surface hollow or (b) substitutional adsorption site. DFT-LDA results of relaxations of the adsorbate and first substrate layer are given relative to the interlayer spacing of the $\mathrm{Cu}$ bulk.

$$
\begin{aligned}
E^{\mathrm{ad}}= & \frac{1}{2}\left(E^{\mathrm{Cu}(001)}-2 N^{\mathrm{Cu}} E^{\mathrm{Cu}-\text { bulk }}+2 N^{\mathrm{Co}} E^{\mathrm{Co}-\text { atom }}\right. \\
& \left.-E^{\mathrm{Cu}(001)-(3 \times 3)-\mathrm{Co}}\right) .
\end{aligned}
$$

Here $E^{\text {Cu-bulk }}$ is the fcc bulk energy of $\mathrm{Cu}$ and $E^{\text {Co-atom }}$ is the energy of the free Co atom. For the on-surface adsorption $\left(N^{\mathrm{Cu}}=0, N^{\mathrm{Co}}=1\right)$, we obtain $E_{\text {on-surf. }}^{\text {ad }}=3.93 \mathrm{eV}$ and for the substitutional adsorption $\left(N^{\mathrm{Cu}}=N^{\mathrm{Co}}=1\right)$, we obtain $E_{\text {sub. }}^{\mathrm{ad}}$. $=5.29 \mathrm{eV}$. In this case the ejected $\mathrm{Cu}$ adatom is assumed to diffuse to a step and be rebound at a kink site. The substitutional adsorption is energetically favored by $1.36 \mathrm{eV}$ over the on-surface adsorption. This is attributed to the higher coordination number of eight substrate atoms for the substitutional adsorption compared to four substrate atoms for the on-surface site. We note in passing that phenomena leading to a surface intermixing in heteroepitaxy have been explained in terms of the surface free energies of the contributing materials. ${ }^{20,23}$ However, in the system investigated here we find that the difference of surface energies would rather lead to a compact cobalt island and eventually to copper capping. ${ }^{29}$ The substitutional adsorption represents an alternative only for low coverages in terms of a local thermal equilibrium, as long as interaction between the different Co adatoms is not effective.

The above results suggest to interpret the indentations in the STM images (see Fig. 1) as atomically resolved single Co atoms incorporated in the substrate surface layer. Thus, in addition to the substitutional cobalt, there are Co as well as $\mathrm{Cu}$ adatoms diffusing on the surface. The consequences of this situation on the growth behavior were studied with STM comparing island size distributions of 0.11-ML Co deposited on the clean $\mathrm{Cu}(001)$ substrate at identical rates (0.2 ML/min) for two different temperatures, $295 \mathrm{~K}$ and $415 \mathrm{~K}$ (Fig. 3). According to the traditional view of nucleation as a result of binary collisions, ${ }^{24}$ a Poisson-like island size distribution with a peak close to the mean island size would be expected. Instead, the size distributions in Fig. 3 exhibit a maximum at very small island sizes and then an unusually gradual decay for larger sizes. Fassbender, Allenspach, and Dürig ${ }^{23}$ note a similar observation: a broad island size distribution yet for higher coverages (0.6 ML) at $330 \mathrm{~K}$. The island densities obtained from the two images (a) and (b) in Fig. 3 are $n_{x}=1.64 \times 10^{-3}$ and $n_{x}=2.23$ $\times 10^{-3}$ islands/surface unit cell, respectively. The deposition of Co at $415 \mathrm{~K}$ results in a higher island density compared to the deposition at room temperature, which is at variance with the classical scenario where $n_{x} \propto \exp \left[E /\left(k_{B} T\right)\right]$. Additionally, Fassbender, Allenspach, and Dürig ${ }^{23}$ found that the island density does not saturate before coalescence sets in. The above experimental findings can be interpreted under the assumption that (i) the Co atoms incorporated in the top substrate layer act as pinning centers for diffusing surface adatoms and (ii) in the initial stage of growth the pinning centers are continuously generated during deposition whereby the exchange process is thermally activated.

Atomic exchange as a mechanism for the creation of nucleation sites has been recently discussed using mean-field rate equations and Monte Carlo simulations..$^{20,30,31}$ In the present work we investigate quantitatively the role of the incorporated cobalt atoms as nucleation centers. We calcu- 
a) $\mathbf{T}=\mathbf{2 9 5 K}$
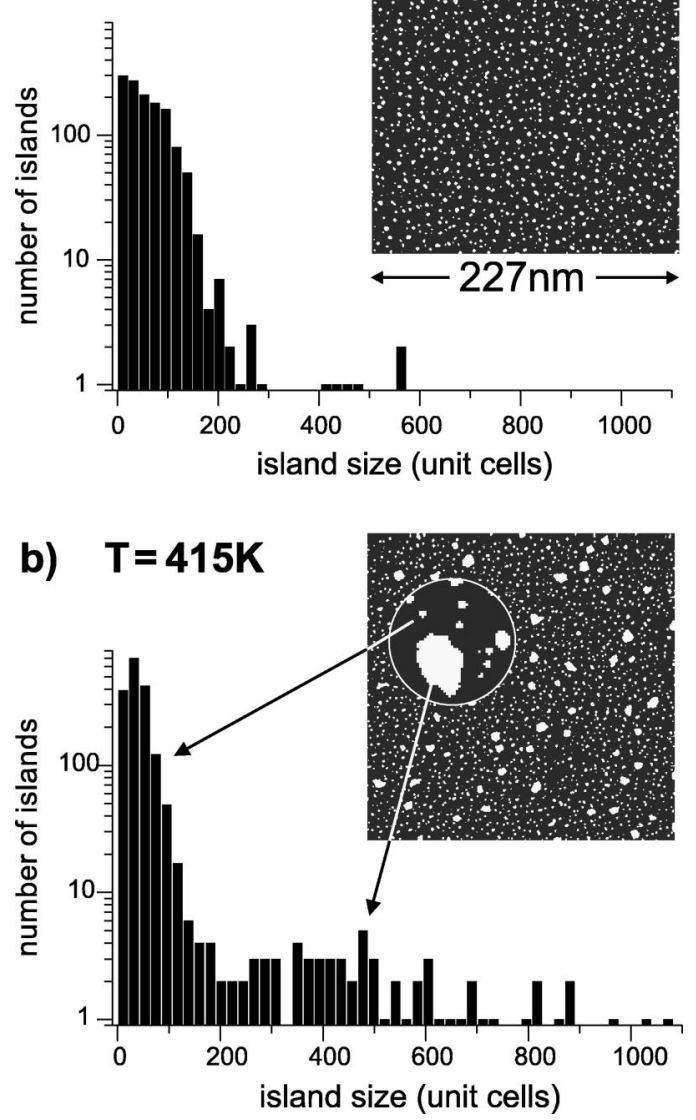

FIG. 3. STM images and their island size distributions of 0.11 ML Co deposited on $\mathrm{Cu}(001)$ with identical deposition rates $(0.2$ $\mathrm{ML} / \mathrm{min}$ ) at (a) $295 \mathrm{~K}$ and (b) $415 \mathrm{~K}$. The bimodal growth mode is clearly visible in (b). As indicated by the arrows, large islands correspond to the broad maximum at about 400 surface unit cells, whereas the sizes of the small islands are concentrated at very low values. The circle in the STM image in (b) represents a magnification.

lated the adsorption energy of $\mathrm{Co}$ and $\mathrm{Cu}$ atoms at hollow sites on the clean $\mathrm{Cu}(001)$ and on the $\mathrm{Cu}(001)$ surface with $(3 \times 3)$ substitutional Co atoms. The isolated adatom on the clean $\mathrm{Cu}(001)$ surface is marked as "far." Depending on the position with respect to the substituted $\mathrm{Co}$ atom, there are two different adsorption sites on the $\mathrm{Cu}(001)-(3 \times 3)$-Co subsurface: one where the adatom $(\mathrm{Co}$ or $\mathrm{Cu})$ is the nearest neighbor and another where it is $1.5 a_{\mathrm{Cu}}^{0}\left(a_{\mathrm{Cu}}^{0}\right.$ lattice constant of bulk $\mathrm{Cu}$ ) away from the substituted Co atom (Fig. 4). Additionally, we estimated the adsorption energies for Co and $\mathrm{Cu}$ adatoms at a kink site of a $\mathrm{Cu}$ step or island. For a $\mathrm{Cu}$ adatom this energy equals the cohesive energy. The kink-site
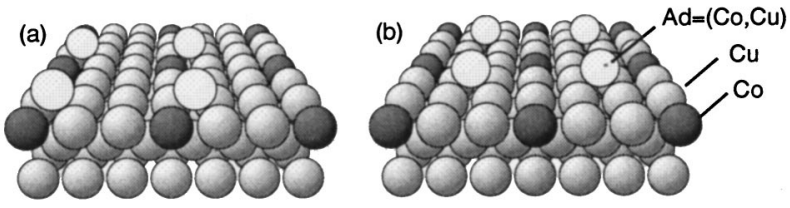

FIG. 4. Structures of $(3 \times 3)$ substitutional $\mathrm{Co}$ on $\mathrm{Cu}(001)$ with additional adatoms $(\mathrm{Co}$ or $\mathrm{Cu})$ at an on-surface hollow site as (a) nearest neighbor or (b) $1.5 a_{\mathrm{Cu}}^{0}$ away from the substituted Co atom.
TABLE I. Calculated adsorption energy changes of $\mathrm{Co}$ and $\mathrm{Cu}$ adatoms at a fcc hollow site on the intermixed $\mathrm{Cu}(001)-(3$ $\times 3$ )-Co subsurface and at a kink site of the $\mathrm{Cu}$ substrate with respect to $E^{\text {ad }}$ for the clean $\mathrm{Cu}(001)$ surface.

\begin{tabular}{lcc}
\hline \hline Adsorption position & $\Delta E_{\mathrm{Cu}}^{\mathrm{ad}}(\mathrm{eV})$ & $\Delta E_{\mathrm{Co}}^{\mathrm{ad}}(\mathrm{eV})$ \\
\hline $1.5 a_{\mathrm{Cu}}^{0}$ & -0.06 & -0.08 \\
Nearest neighbor & 0.16 & 0.26 \\
Kink site at a $\mathrm{Cu}$ island & 0.82 & 1.25 \\
\hline \hline
\end{tabular}

energy of a Co adatom was obtained by a least-squares fit of the energy as a function of the local coordination number $Z_{I}: 32,33 E=\Sigma_{I} E_{I}$ with $E_{I}=E_{I}^{0}+A_{I} \sqrt{Z_{I}}+B_{I} Z_{I}$, where $E_{I}^{0}$ is the energy of a free atom with $I=(\mathrm{Cu}, \mathrm{Co})$. The bulk, the clean $\mathrm{Co}(001)$ and $\mathrm{Cu}(001)$ surfaces, and the adsorbate systems discussed above were considered in the fit. ${ }^{34}$

The adsorption energies from DFT calculations and the kink-site energies obtained with the coordination number model are listed in Table I as energy changes with respect to the ones of the isolated adatoms ("far") on $\mathrm{Cu}(001)$ $\left(E_{\mathrm{Cu} \text { far }}^{\mathrm{ad}}=3.68 \mathrm{eV}, E_{\mathrm{Co} \text { far }}^{\mathrm{ad}}=3.95 \mathrm{eV}\right)$. The first striking fact is that the Co adatom is always bound stronger on the surface than the $\mathrm{Cu}$ adatom due to a stronger $d-d$ and $d-s p$ hybridization. The higher adsorption energy of Co is responsible for the lower mobility of $\mathrm{Co}$ on the $\mathrm{Cu}(001)$ surface. Furthermore, we see that the adsorption energies of the isolated adatom and the one at $1.5 a_{\mathrm{Cu}}^{0}$ from the substituted Co are close, so the adatoms are practically not sensitive to the environment going beyond second nearest neighbors. Comparing the two adsorption sites on the substituted surface, we conclude that the incorporated Co atoms indeed act as pinning centers both for the $\mathrm{Co}$ and $\mathrm{Cu}$ atoms, the effect being stronger for $\mathrm{Co}(0.34 \mathrm{eV})$ than for $\mathrm{Cu}(0.22 \mathrm{eV})$. Consequently, the exchanged $\mathrm{Cu}$ atom needs only about $0.2 \mathrm{eV}$ to overcome the attractive potential of the substituted Co and diffuse as adatom on the surface. Considering the higher mobility of $\mathrm{Cu}$ and the energy gain at a kink site, the $\mathrm{Cu}$ adatom is likely to be rebound at a kink site of a $\mathrm{Cu}$ step or a $\mathrm{Cu}$ island. This suggests strongly that although Co is being deposited in the initial stage of growth the first stable islands are formed essentially by $\mathrm{Cu}$ adatoms. Experimental evidence for this will be discussed below.

With increasing coverage the binding of deposited Co at the previously generated pinning centers as well as the aggregation of deposited $\mathrm{Co}$ at the $\mathrm{Cu}$ islands will occur as processes competing with the atomic exchange. Moreover, as mentioned above, total-energy calculations show that with increasing Co coverage the substitutional Co adsorption gets less favorable than the formation of compact islands. ${ }^{29}$ Two consequences emerge from this: (i) high nucleation density of Co islands and (ii) Co decoration of the $\mathrm{Cu}$ islands at elevated temperatures.

These theoretical predictions were indeed confirmed in a CO-titration experiment. AES test measurements of COexposed surfaces at $T=295 \mathrm{~K}$ clearly showed strong $\mathrm{CO}$ adsorption on a $\mathrm{Co}(001)$ surface [5 ML grown on $\mathrm{Cu}(001)$ ] and negligible adsorption on the pure $\mathrm{Cu}(001)$ surface. It is therefore possible to correlate $\mathrm{CO}$ adsorption induced features in the STM images with Co sites at the surface. This direct identification has clear advantages compared to iden- 


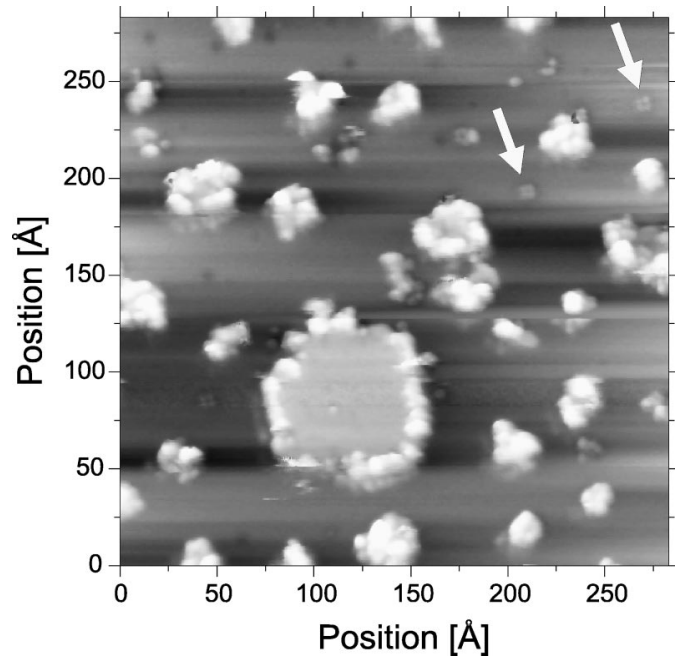

FIG. 5. STM image $\left(U_{\text {tip }}=3.5 \mathrm{~V}, I=0.4 \mathrm{nA}\right) \quad$ of $0.11 \mathrm{ML} \mathrm{Co} / \mathrm{Cu}(001)$ deposited at $415 \mathrm{~K}$ and afterwards exposed to about $20 \mathrm{~L} \mathrm{CO}$. Adsorption induced features occur on the small islands, on the edges of the large islands, and at atomic-size indentations (white arrows).

tification via bias voltage dependent contrast inversion used in Ref. 23. The latter is based on the electronic structure of tip and sample and thus does not permit an unambiguous interpretation. The STM image in Fig. 5 corresponds to a deposition of 0.11 ML Co at $T=415 \mathrm{~K}$ followed by an exposure to $20 \mathrm{CO}$ at $T=295 \mathrm{~K}$. The bright clouds are observed only after the exposure and are attributed to the adsorbed CO molecules. The small islands are nearly completely covered with $\mathrm{CO}$, while on the large islands $\mathrm{CO}$ is adsorbed only at the edges proving that the small islands consist mainly of $\mathrm{Co}$ and the large ones of $\mathrm{Cu}$ with $\mathrm{Co}$ decoration at the edges.
Indeed, a close inspection of the STM image for $415 \mathrm{~K}$ in Fig. 3(b) and the corresponding island size distribution reveals two different kinds of islands, which can be identified by their mean sizes. In addition to a large amount of small islands, a few much larger islands are represented in the island size distribution by a broad maximum at an island area of about 400 surface unit cells. The significantly larger separation between the large islands compared to the separation between the small ones correlates with the higher mobility of the $\mathrm{Cu}$ atoms on the surface-and supports the above quantitative evidence that the large islands consist mainly of $\mathrm{Cu}$. A series of STM images, recorded for various coverages at elevated temperatures, clearly revealed that the large islands grow first before the small islands nucleate in between. ${ }^{35}$ Generally, two microscopic mechanisms initiate the bimodal behavior: exchange mediated nucleation (Co pinning centers) and growth of the $\mathrm{Cu}$ islands. Both island size distributions in Figs. 3(a) and 3(b) result from these mechanisms. Yet, the features of the bimodal growth mode are particularly distinct at $T=415 \mathrm{~K}$ because the processes leading to it are thermally activated as discussed above. We would like to point out that the island size distributions at room temperature are qualitatively very similar to those expected from the exchange mediated growth scenario. ${ }^{31}$

In summary, the results from island size distributions, titration experiments, and $a b$ initio calculations give evidence for a bimodal initial growth mode of $\mathrm{Co}$ on $\mathrm{Cu}(001)$. This growth mode gives rise to formation of large $\mathrm{Cu}$ islands and a high density of small Co islands and is a result of the substitutional adsorption during the initial deposition of Co on $\mathrm{Cu}(001)$.

The work was supported partly by the DFG through SFB 290 and SFB 341 and by the "Innovationsprogramm Nanowissenschaften" of the MSWWF-NRW. We thank W. Peterßen for technical assistance.
${ }^{1}$ Ultrathin Magnetic Structures I and II, edited by B. Heinrich and J.A.C. Bland (Springer-Verlag, Berlin, 1994).

${ }^{2}$ U. Gradmann, in Handbook of Magnetic Materials, edited by K.H.J. Buschow (Elsevier, New York, 1993), Vol. 7, p. 1.

${ }^{3}$ M.N. Baibich, J.M. Broto, A. Fert, F. Ngyen Van Dau, F. Petroff, P. Etienne, G. Creuzet, A. Friedrich, and J. Chazelas, Phys. Rev. Lett. 61, 2472 (1988).

${ }^{4}$ G. Binasch, P. Grünberg, F. Saurenbach, and W. Zinn, Phys. Rev. B 39, 4828 (1989).

${ }^{5}$ S.S.P. Parkin, N. More, and K.P. Roche, Phys. Rev. Lett. 64, 2304 (1990).

${ }^{6}$ P. Grünberg, R. Schreiber, Y. Pang, M.B. Brodsky, and H. Sowers, Phys. Rev. Lett. 57, 2442 (1986).

${ }^{7}$ M.T. Johnson, S.T. Purcell, N.W.E. McGee, R. Coehoorn, J. aan de Stegge, and W. Hoving, Phys. Rev. Lett. 68, 2688 (1992).

${ }^{8}$ P. Bruno, Phys. Rev. B 52, 411 (1995).

${ }^{9}$ P. Zahn, J. Binder, I. Mertig, R. Zeller, and P.H. Dederichs, Phys. Rev. Lett. 80, 4309 (1998).

${ }^{10}$ H. Li and B.P. Tonner, Surf. Sci. 237, 141 (1990).

${ }^{11}$ C.M. Schneider, P. Bressler, P. Schuster, J. Kirschner, J.J. de Miguel, and R. Miranda, Phys. Rev. Lett. 64, 1059 (1990).

${ }^{12}$ P. Krams, F. Lauks, R.L. Stamps, B. Hillebrands, and G.
Güntherodt, Phys. Rev. Lett. 69, 3674 (1992).

${ }^{13}$ A.K. Schmid and J. Kirschner, Ultramicroscopy 42-44, 483 (1992).

${ }^{14}$ W. Weber, A. Bischof, R. Allenspach, C. Wursch, C.H. Back, and D. Pescia, Phys. Rev. Lett. 76, 3424 (1996).

${ }^{15}$ W. Weber, A. Bischof, R. Allenspach, C. H. Back, J. Fassbender, U. May, B. Schirmer, R.M. Jungblut, G. Güntherodt, and B. Hillebrands, Phys. Rev. B 54, 4075 (1996).

${ }^{16}$ M. Hansen, Constitution of Binary Alloys (McGraw-Hill, New York, 1958).

${ }^{17}$ A. Schmalz, S. Aminpirooz, L. Becker, J. Haase, J. Neugebauer, M. Scheffler, D.R. Batchelor, D.L. Adams, and E. Bøgh, Phys. Rev. Lett. 67, 2163 (1991); C. Stampfl and M. Scheffler, Surf. Rev. Lett. 2, 317 (1995).

${ }^{18}$ J. Shen, J. Giergiel, A.K. Schmid, and J. Kirschner, Surf. Sci. 328, 32 (1995).

${ }^{19}$ T. Flores, M. Hansen, and M. Wuttig, Surf. Sci. 371, 1 (1997).

${ }^{20}$ D.D. Chambliss and K.E. Johnson, Phys. Rev. B 50, 5012 (1994).

${ }^{21}$ S. Blügel, Appl. Phys. A: Mater. Sci. Process. 63, 595 (1996).

${ }^{22}$ J.R. Cerda, P.L. deAndres, A. Cebollada, R. Miranda, E. Navas, P. Schuster, C.M. Schneider, and J. Kirschner, J. Phys.: Condens. Matter 5, 2055 (1993). 
${ }^{23}$ J. Fassbender, R. Allenspach, and U. Dürig, Surf. Sci. Lett. 383, L742 (1997).

${ }^{24}$ J.A. Venables, in The Chemical Physics of Solid Surfaces, edited by D.A. King and D.P. Woodruff (Elsevier Science, Amsterdam, 1997), Vol. 8, and references therein.

${ }^{25}$ K. Besocke, Surf. Sci. 181, 145 (1987).

${ }^{26}$ P. Blaha, K. Schwarz, P. Sorantin, and S.B. Trickey, Comput. Phys. Commun. 59, 399 (1990); B. Kohler, S. Wilke, M. Scheffler, R. Kouba, and C. Ambrosch-Draxl, ibid. 94, 31 (1996); M. Petersen, F. Wagner, L. Hufnagel, and M. Scheffler, ibid. (to be published).

${ }^{27}$ J.P. Perdew and Y. Wang, Phys. Rev. B 45, 13244 (1992).

${ }^{28}$ For example, the energy difference of the two investigated adsorption geometries, on surface and substitutional changes by less than $0.1 \mathrm{eV}$ for a $(2 \times 2)$-unit cell if spin polarization is considered: $\Delta E=1.32 \mathrm{eV}$ for a nonmagnetic calculation compared to $\Delta E=1.24 \mathrm{eV}$ for a ferromagnetic calculation. Thus, the conclusions derived from the DFT calculations are not affected by magnetism.

${ }^{29}$ R. Pentcheva and M. Scheffler (unpublished).

${ }^{30}$ J.A. Meyer and R.J. Behm, Surf. Sci. 322, L275 (1995).

${ }^{31}$ A. Zangwill and E. Kaxiras, Surf. Sci. 326, L483 (1995).

${ }^{32}$ D. Spanjaard and M.C. Desjonquéres, in Interaction of Atoms and Molecules with Solid Surfaces, edited by V. Bortolani (Plenum, New York, 1990).

${ }^{33}$ M. Methfessel, D. Henning, and M. Scheffler, Appl. Phys. A: Solids Surf. 55, 442 (1992).

${ }^{34} E_{\mathrm{Cu}}^{0}=-3275.548 \mathrm{Ry}, E_{\mathrm{Co}}^{0}=-2760.381 \mathrm{Ry}$. From a leastsquares fit to various DFT-LDA results we obtained: $A_{\mathrm{Cu}}$ $=-0.105 \mathrm{Ry}, A_{\mathrm{Co}}=-0.090 \mathrm{Ry}, B_{\mathrm{Cu}}=0.003 \mathrm{Ry}$, and $B_{\mathrm{Co}}$ $=-0.012$ Ry.

${ }^{35}$ F. Nouvertné, U. May, U. Korte, and G. Güntherodt (unpublished). 